\title{
Malaria Diagnosed in an Urban Setting Strongly Associated with Recent Overnight Travel: A Case-Control Study from Kampala, Uganda
}

Emmanuel Arinaitwe, ${ }^{1,2 \star}$ Arthur Mpimbaza, ${ }^{2,3}$ Joaniter I. Nankabirwa, ${ }^{2,4}$ Victor Kamya, ${ }^{2}$ Alan Asiimwe, ${ }^{2}$ Julius K. Kuule, ${ }^{5}$ Moses R. Kamya, ${ }^{2,4}$ Chris Drakeley, ${ }^{1}$ Grant Dorsey, ${ }^{6}$ Philip J. Rosenthal, ${ }^{6}$ and Sarah G. Staedke ${ }^{1}$

${ }^{1}$ London School of Hygiene and Tropical Medicine, London, United Kingdom; ${ }^{2}$ Infectious Diseases Research Collaboration, Kampala, Uganda; ${ }^{3}$ Child Health and Development Centre, Makerere University College of Health Sciences, Kampala, Uganda; ${ }^{4}$ Department of Medicine, Makerere University, Kampala, Uganda; ${ }^{5}$ Uganda Malaria Research Centre, Ministry of Health, Kampala, Uganda; ${ }^{6}$ Department of Medicine, University of California, San Francisco, California

\begin{abstract}
Malaria is frequently diagnosed in urban Kampala, despite low transmission intensity. To evaluate the association between recent travel out of Kampala and malaria, we conducted a matched case-control study. Cases were febrile outpatients with a positive malaria test; controls were febrile outpatients with a negative test. For every two cases, five controls were selected, matching on age. Data were collected on recent overnight travel out of Kampala (past 60 days), destination and duration of travel, and behavioral factors, including sleeping under an insecticide-treated net (ITN) during travel. From July to August 2019, 162 cases and 405 controls were enrolled. The locations of residence of cases and controls were similar. More controls were female $(62.7 \%$ versus $46.3 \%, P<0.001)$. Overall, $158(27.9 \%)$ participants reported recent overnight travel. Travelers were far more likely to be diagnosed with malaria than those who did not travel (80.4\% versus 8.6\%, OR 58.9, 95\% Cl: 23.1-150.1, $P<0.001)$. Among travelers, traveling to a district not receiving indoor residual spraying of insecticide (OR 35.0, 95\% Cl: $4.80-254.9, P<0.001)$, no ITN use (OR 30.1, 95\% Cl: $6.37-142.7, P<0.001$ ), engaging in outdoor activities (OR 22.0, 95\% Cl: 3.42-141.8, $P=0.001$ ), and age $<16$ years (OR 8.36, 95\% Cl: 2.22-56.2, $P=0.03$ ) were associated with increased odds of malaria. Kampala residents who traveled overnight out of the city were at substantially higher risk of malaria than those who did not travel. For these travelers, personal protection measures, including sleeping under an ITN when traveling, should be advocated.
\end{abstract}

\section{INTRODUCTION}

Over the past 20 years, impressive reductions in malaria burden have been reported in across sub-Saharan Africa. ${ }^{1}$ This progress has been attributed to high coverage of effective interventions, including indoor residual spraying (IRS) of insecticides, long-lasting insecticidal bed nets, and prompt treatment with artemisinin-based combination therapy. Increasing urbanization of African populations has also contributed to malaria reduction. ${ }^{2}$ Despite this success, malaria remains a global health challenge; recent evidence indicates that the number of malaria cases in many African countries has increased, suggesting that progress on malaria control has stalled. ${ }^{3}$ In Uganda, expansion of malaria control measures has been associated with substantial decreases in malaria burden in some areas, ${ }^{4-6}$ but not in all settings. ${ }^{7,8}$ Consequently, malaria transmission in Uganda is heterogenous, with low risk of malaria in urban areas, highland areas, and locations where IRS has been effectively implemented, but medium to high risk elsewhere in Uganda. ${ }^{9}$

In residents of malaria-endemic areas, repeated exposure to malaria parasites results in acquisition of protective immunity against illness and infection. ${ }^{10-12}$ However, this acquired immunity may wane if exposure to malaria parasites reduces because of a non-endemic area or highly urbanized environment or in the setting of intense malaria control measures. ${ }^{13}$ In Zanzibar, Tanzania, and Bioko Island, Equatorial Guinea, travel between the island (low malaria risk following scale-up of control measures) and the mainland (high malaria risk) was found to be associated with malaria infection and

*Address correspondence to Emmanuel Arinaitwe, London School of Hygiene and Tropical Medicine, Keppel Street, London, WC1E 7HT, United Kingdom and Infectious Diseases Research Collaboration, 2C Nakasero Hill Road, P.O. Box 7475, Kampala, Uganda. E-mails: earinaitwe@idrc-uganda.org or arinaitwe.emmanuel@lshtm.ac.uk disease among returning residents. ${ }^{14-18}$ In Malawi, among travelers between urban and rural areas, symptomatic malaria was higher in participants living in urban zones who reported spending at least one night in rural areas within a month before testing for malaria. ${ }^{19}$ In Tororo District in Eastern Uganda, where an effective IRS campaign resulted in significant declines in malaria transmission intensity, ${ }^{20}$ travel from Tororo to neighboring high-transmission settings was a risk factor for malaria. $^{21}$

The WHO recommends malaria chemoprophylaxis for those traveling from non-endemic regions to malaria-endemic areas. ${ }^{22}$ However, whether this policy or other preventative approaches would be applicable for travel within malariaendemic countries is less clear. In Uganda, malaria transmission intensity appears to have decreased greatly in Kampala, the urban capital, but the number of malaria cases reported in Kampala fluctuates over time. ${ }^{23}$ To investigate the association between overnight travel outside of Kampala and diagnosis of clinical malaria, we conducted a case-control study.

\section{METHODS}

Study site. The study was conducted at Naguru General Hospital, a public hospital in Kampala. Kampala is a low malaria transmission setting, with a reported parasite prevalence rate in children aged $0-59$ months of $3.7 \%$ by histidinerich protein 2 (HRP-2) rapid diagnostic test (RDT) in 2014-2015. ${ }^{9}$ The hospital serves the Kampala metropolitan area and provides outpatient, inpatient, and specialized care services. Between January and September 2019, the outpatient department served an average of 13,000 patients per month, out of which 1,000 were referred to the laboratory for a malaria test, and an average of 110 tested positive for malaria per month. Malaria was typically tested using RDT, with 
quantification of parasite density by microscopy for positive RDT tests. By policy, all patients with suspected malaria were referred to the laboratory for a malaria test before malaria treatment was prescribed.

Study design. This was a matched case-control study. Cases were defined as patients at least 6 months of age attending the outpatient department at Naguru General Hospital with documented axillary temperature of $\geq 37.5^{\circ} \mathrm{C}$ and/or history of fever within the last 24 hours with a positive RDT for malaria. Controls were patients attending the same outpatient department with documented axillary temperature of $\geq 37.5^{\circ} \mathrm{C}$ and/or history of fever within the last 24 hours, but with a negative malaria RDT.

Matching criteria. Controls were matched to cases by age and time of presentation (within 1 week of a case). For every two malaria-positive cases, five malaria-negative controls were enrolled. Age categories matched were $0.5-5$ years, $>5-15$ years, and $>15$ years.

Study participant enrollment and data collection. All patients referred to the outpatient department laboratory for suspected malaria were assessed for eligibility to participate in the study. Study participants were enrolled if they 1) resided in Kampala (Kampala Central, Kawempe, Makindye, Nakawa, and Rubaga divisions) for at least the last 3 months, 2) were at least 0.5 years of age, 3) weighed at least $4 \mathrm{~kg}$, 4) had axillary temperature of at least $37.5^{\circ} \mathrm{C}$ and/or a history of fever within the last 24 hours, 5) were able to speak English or Luganda, and 6) provided written informed consent (from parents or guardians in children, with assent for ages 8-17 years). Patients with a positive malaria RDT result were screened as cases and patients with a negative malaria RDT result were screened as controls until 162 cases and 405 controls were enrolled.

For patients who met all the eligibility criteria and provided informed consent, a questionnaire was administered on whether they had traveled out of Kampala within the last 60 days. If they had traveled, a detailed travel questionnaire was administered, to capture data on destination of travel, length of the trip, and behavioral risk factors likely to influence exposure to mosquito bites such as staying outdoors late, time to bed, and use of insecticide-treated nets (ITNs) during travel.

Laboratory procedures. Malaria was diagnosed at Naguru General Hospital by experienced laboratory technicians with the CareStart ${ }^{T M}$ RDT (Access Bio Inc., Somerset, NJ), which assesses HRP-2, according to the manufacturer's instructions.

Sample size. We aimed to detect an association between recent (within 60 days) overnight travel out of Kampala and diagnosis of malaria. To do this, a sample size of 567 participants was required to detect an odds ratio of at least 3.0 for the association between overnight travel and malaria diagnosis, given a two-sided type 1 error of 0.05 and power of $80 \%$. We predicted that $56 \%$ of cases and $23 \%$ of controls would report recent overnight travel based on data from a peri-urban area in Jinja, Uganda, ${ }^{21}$ and thus 162 cases and 405 controls (ratio of 2:5) were required to evaluate the association between risk factors for malaria and overnight travel.

Ethical considerations. Ethical approvals were obtained from the Mulago Hospital Research and Ethics Committee (MHREC; ref. 1592), the London School of Hygiene and Tropical Medicine Ethics Committee (LSHTM; ref. 16625), and the Uganda National Council of Science and Technology (UNCST; ref. SS 5012). Informed consents were obtained from all study participants before data collection. All study staff were trained on procedures for maintaining confidentiality before study activities commenced.

Statistical analysis. All data were collected by trained study staff using a hand-held computer and transferred weekly to a secure server. Data analyses were performed using Stata 14 software (StataCorp, College Station, TX). Recent overnight travel was defined as any travel outside the geographical confines of Kampala, spending at least one night away, within the previous 60 days. A 60-day threshold was chosen to represent the period when most newly acquired malaria infections were likely to become symptomatic, based on the results of a prior study conducted in Uganda. ${ }^{21}$ For study participants with a history of recent overnight travel, destination of travel was dichotomized into districts receiving or not receiving IRS according to U.S. President's Malaria Initiative VectorLink, Uganda IRS project. ${ }^{24}$ Other risk factors that were evaluated included duration of stay at destination of travel, reported ITN use, time to bed, place of stay, and activities carried out during travel. Malaria cases diagnosed within 7 days from the start of a trip were not included in the analysis because the incubation period of Plasmodium falciparum malaria typically ranges from 7 to 14 days depending on the immune status of the individual. ${ }^{25-27}$ Characteristics of study participants and individual trips were reported as proportions for categorical variables and medians with interquartile ranges for continuous variables.

The primary exposure of interest was recent overnight travel. Conditional logistic regression analysis was used to estimate associations (as odds ratios) between recent overnight travel out of Kampala and diagnosis of malaria. Because the analysis was matched on age, association between age and the odds of malaria was not reported. For study participants with a history of recent overnight travel, unmatched analysis of risk factors associated with malaria during travel out of Kampala was performed using logistic regression, with associations reported as odds ratios. Interactions among risk factors of malaria during travel were explored, and none were found to be statistically significant. Reasons why participants traveled were not included in the final model analysis because of collinearity of some reasons, for example, accompanying parents or guardians with age categories. All comparisons were considered statistically significant at $P<0.05$.

\section{RESULTS}

Characteristics of study participants. From July through August 2019, 186 cases and 524 controls were screened, and 162 cases and 405 controls were enrolled (Figure 1). All cases and most controls were excluded because they resided outside of Kampala. Cases and controls were similar in age and location of residence, but not gender (Table 1); more controls than cases were female $(62.7 \%$ versus $46.3 \%, P<0.001)$. Overall, 158 participants reported recent overnight travel, including 128 (78.4\%) cases and 31 (7.7\%) controls.

Characteristics of participants who traveled. Of those participants who traveled (Table 2), more cases than controls were aged $<5$ years $(33.1 \%$ versus $9.7 \%, P<0.001)$. Participants made only one trip within 60 days and traveled to all regions across the country, although they traveled to districts 


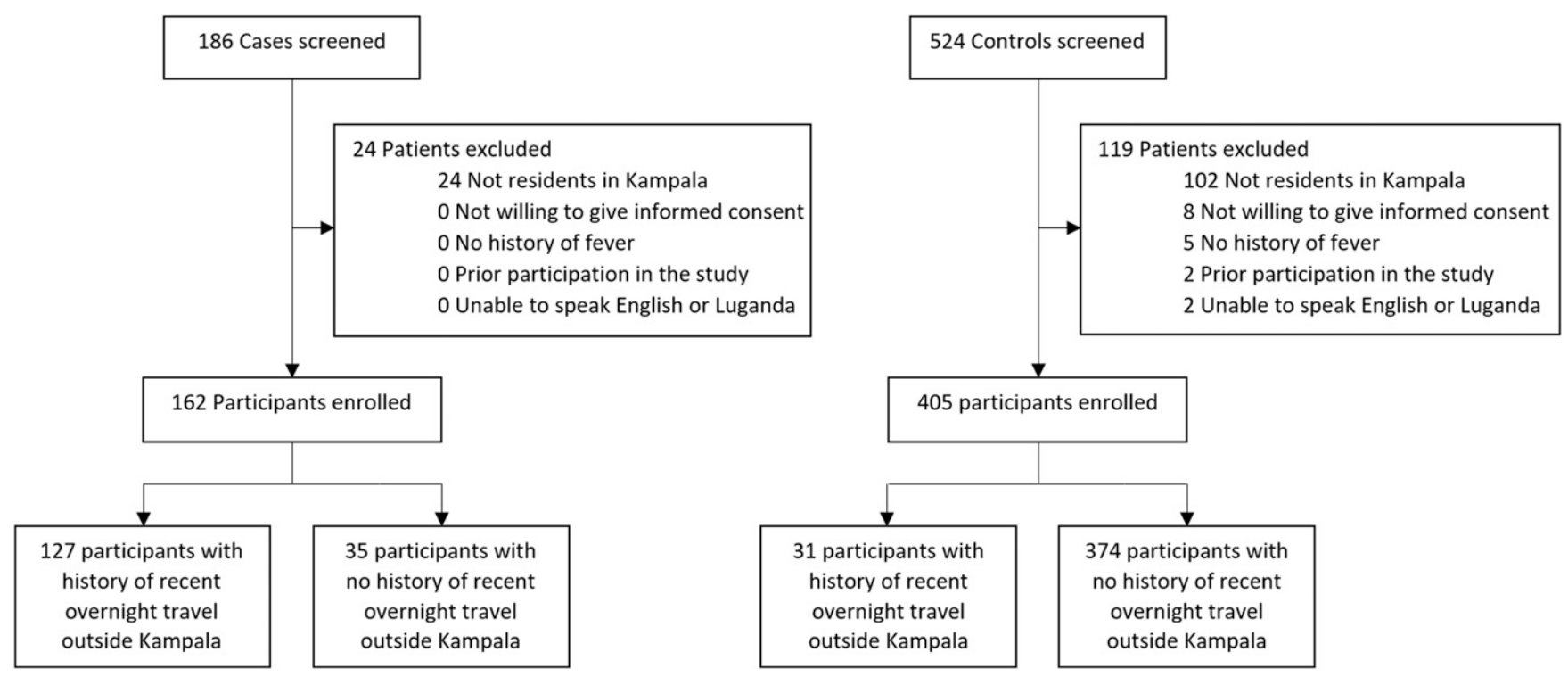

FIGURE 1. Study profile.

neighboring Kampala more frequently (Figure 2). For both groups, the median duration of stay when traveling was 3 days. Compared with controls, cases were more likely to have traveled to a district without an ongoing IRS program (97.6\% versus $67.7 \%, P<0.001)$. Both cases and controls commonly traveled to visit relatives and for funerals, but controls were more likely to travel for work (19.4\% versus $3.9 \%, P=0.02)$. Travelers in both groups commonly stayed in the home of friends or relatives and engaged in similar evening activities, although cases were more likely to sit outside in the garden (23.6\% versus $6.3 \%, P<0.001)$. Controls were far more likely than cases to have slept under an ITN while traveling $(64.5 \%$ versus $15.8 \%, P<0.001)$.

Association between recent overnight travel outside Kampala and malaria. In an analysis adjusted for gender and location of residence (Table 3), recent overnight travel was strongly associated with malaria; participants who traveled within the last 60 days had significantly higher odds of a positive malaria test than those without recent travel $(80.4 \%$ versus $8.6 \%$, adjusted odds ratio [aOR] $58.9,95 \% \mathrm{Cl}$ :
23.1-150.1, $P<0.001)$. The odds of malaria was also higher in males than in females (36.6\% versus $22.8 \%$, aOR $2.0,95 \% \mathrm{Cl}$ : 1.02-3.91, $P=0.04$ ). No association between location of residence and malaria was found (Table 3).

Factors associated with malaria among participants who traveled. In an analysis restricted to participants who traveled, older age, travel to a non-IRS district, duration of travel for 10 days or more, not using an ITN during travel, staying with friends and relatives, and engaging in outdoor evening activities were all associated with malaria (Table 4). Participants who traveled to a nonIRS district were at much higher odds of malaria than those who traveled to a district covered by IRS (85.5\% versus $23.1 \%$, OR 35.0, 95\% Cl: 4.8-254.9, $P<0.001$ ) as were participants who did not use an ITN when traveling, compared with those who did (90.7\% versus 50.0\%, OR 30.1, 95\% Cl: $6.4-142.7, P<0.001)$.

\section{DISCUSSION}

Our findings from a matched case-control study indicate that travel out of Kampala was strongly associated with increased

TABLE 1

Characteristics of study participants

\begin{tabular}{lcc}
\hline \multicolumn{1}{c}{ Characteristic } & Cases (positive malaria test) & Controls (negative malaria test) \\
\hline Total number of study participants $(N=567)$ & 162 & 405 \\
Mean age (SD), years & $17.7(16.3)$ & $18.6(17.3)$ \\
Age categories, $n(\%)$ & $56(34.6)$ & $140(34.6)$ \\
$\quad$ Below 5 years & $26(16.1)$ & $65(16.1)$ \\
5-15 years & $80(49.4)$ & $200(49.4)$ \\
16 years or older & $75(46.3)$ & $254(62.7)$ \\
Female gender, $n(\%)$ & & $37(9.1)$ \\
Division of residence in Kampala, $n(\%)$ & $11(6.8)$ & $21(5.2)$ \\
Kampala central & $15(9.3)$ & $41(10.1)$ \\
Kawempe & $20(12.4)$ & $295(72.8)$ \\
Makindye & $114(70.4)$ & $11(2.7)$ \\
Nakawa & $2(1.2)$ & $31(7.7)$ \\
Rubaga & $127(78.4)$ & \\
Participants with history of ovemight travel, $n(\%)$ & & \\
\hline
\end{tabular}


TABLE 2

Characteristics of participants with a history of recent overnight travel

\begin{tabular}{|c|c|c|}
\hline Characteristic & Cases (positive malaria test) & Controls (negative malaria test) \\
\hline \multicolumn{3}{|l|}{ Age categories, $n(\%)$} \\
\hline Below 5 years & $42(33.1)$ & $3(9.7)$ \\
\hline $5-15$ years & $18(14.2)$ & 0 \\
\hline 16 years or older & $67(52.8)$ & $28(90.3)$ \\
\hline Female gender, $n(\%)$ & $60(47.2)$ & 19 (61.3) \\
\hline Duration of stay, median (IQR), d & $3(2-9)$ & $3(2-6)$ \\
\hline Travel to non-IRS district ${ }^{\star}, n(\%)$ & $124(97.6)$ & $21(67.7)$ \\
\hline $\begin{array}{l}\text { Time from first night of travel to } \\
\text { presentation with fever, median (IQR), day }\end{array}$ & $22(13-45)$ & $24(7-44)$ \\
\hline \multicolumn{3}{|l|}{ Primary reason for overnight travel, $n(\%)$} \\
\hline Visiting relatives & $58(45.7)$ & $19(61.3)$ \\
\hline Accompanying parents & $33(26.0)$ & $1(3.2)$ \\
\hline Funeral rite & $18(14.2)$ & $3(9.7)$ \\
\hline Work & $5(3.9)$ & $6(19.4)$ \\
\hline Attending school & $5(3.9)$ & 0 \\
\hline Conference or workshop & $5(3.9)$ & 0 \\
\hline Party or cultural gathering & $3(2.4)$ & $2(6.5)$ \\
\hline \multicolumn{3}{|l|}{ Primary place for accommodation, $n(\%)$} \\
\hline Friend's or relative's home & $108(85.0)$ & $26(83.9)$ \\
\hline Camp & $10(7.9)$ & $2(6.5)$ \\
\hline School & 5 (3.9) & 0 \\
\hline Hotel & $2(1.6)$ & $3(9.7)$ \\
\hline Hospital & $1(0.8)$ & 0 \\
\hline Church & $1(0.8)$ & 0 \\
\hline \multicolumn{3}{|l|}{ Primary activity in the evening, $n(\%)$} \\
\hline Went to bed right away & $42(33.1)$ & $6(19.4)$ \\
\hline Watched TV/listened to the radio & $36(28.4)$ & $17(54.8)$ \\
\hline Sat in the gardens & $30(23.6)$ & $2(6.3)$ \\
\hline Stayed at work & $9(7.1)$ & 0 \\
\hline Had a drink with friends & $9(7.1)$ & 0 \\
\hline Helped with house chores & $1(0.8)$ & $6(19.4)$ \\
\hline Went to bed after 9 PM, $n(\%)$ & $84(66.1)$ & $25(80.7)$ \\
\hline Slept under insecticide-treated net, $n$ (\%) & $20(15.8)$ & $20(64.5)$ \\
\hline
\end{tabular}

IRS = indoor residual spraying.

*District that has not received IRS within 12 months before travel.

odds of clinical malaria for Kampala residents presenting with fever. Among travelers, failure to sleep under an ITN during travel and travel to districts that had not received IRS were strongly associated with increased risk of malaria. Other factors associated with increased odds of malaria in travelers included engaging in outdoor activities, age below 16 years, travel for $\geq 10$ days, and staying at a relative's home. According to the Uganda malaria indicator survey in 2018-2019, the proportion of children under 5 years of age who tested positive for malaria in Kampala was much lower ( $0.2 \%$ by microscopy and $1.6 \%$ by RDT) than in the rest of the country ( $9.1 \%$ by microscopy and $16.9 \%$ by RDT). ${ }^{28}$ These findings suggest that Kampala residents who are diagnosed with malaria are likely to have acquired their infection because of recent overnight travel outside of the city, and personal protection measures such as sleeping under an ITN should be emphasized to prevent malaria infection during travel.

We found that many study participants (27.9\%), all residents of Kampala presenting to Naguru General Hospital with fever, had a history of recent overnight travel. Our findings were consistent with a prior study conducted at three sites of varied malaria transmission intensity in Uganda, in which $23.3 \%$ of participants living in a periurban setting had traveled out of their subcounty of residence, with a greater than 3-fold increase in the incidence of malaria following overnight travel. ${ }^{21}$ Our findings are also consistent with those from additional studies from other
African countries. ${ }^{19,29}$ A case-control study conducted in urban and peri-urban Malawi suggested that overnight travel increased the risk of malaria. ${ }^{19}$ Additional studies conducted in Africa showed that returning residents, defined as residents of a low-risk area returning from a trip away, were at a high risk of malaria. ${ }^{14,18,30}$ In Zanzibar and Bioko Island, parasites were introduced by returning residents, which frustrated malaria control efforts. ${ }^{14,18}$ Similarly, in Swaziland and the regions of KwaZulu-Natal, Mpumalanga, and Limpopo in South Africa, malaria parasites were reintroduced from Mozambique following implementation of intensive malaria control. ${ }^{30,31}$ These findings from malaria-endemic areas in Africa highlight that traveling between low and high malaria transmission areas is common, and that returning residents are at increased risk of malaria.

In study participants who traveled, we found that ITN use was uncommon and strongly associated with an increased risk of malaria. Previous studies have also suggested that ITN use was reduced during overnight travel. ${ }^{21,32}$ Reasons for infrequent use of ITNs included having no place to hang the ITN, limited availability of ITNs, and social barriers, such as fear of appearing disrespectful to others. ${ }^{32}$ As malaria control interventions are scaled-up, it is important to encourage use of ITNs during travel to areas of high malaria risk. Policy makers should also consider increasing ITN availability so that those at risk, whether travelers or residents, are protected. 


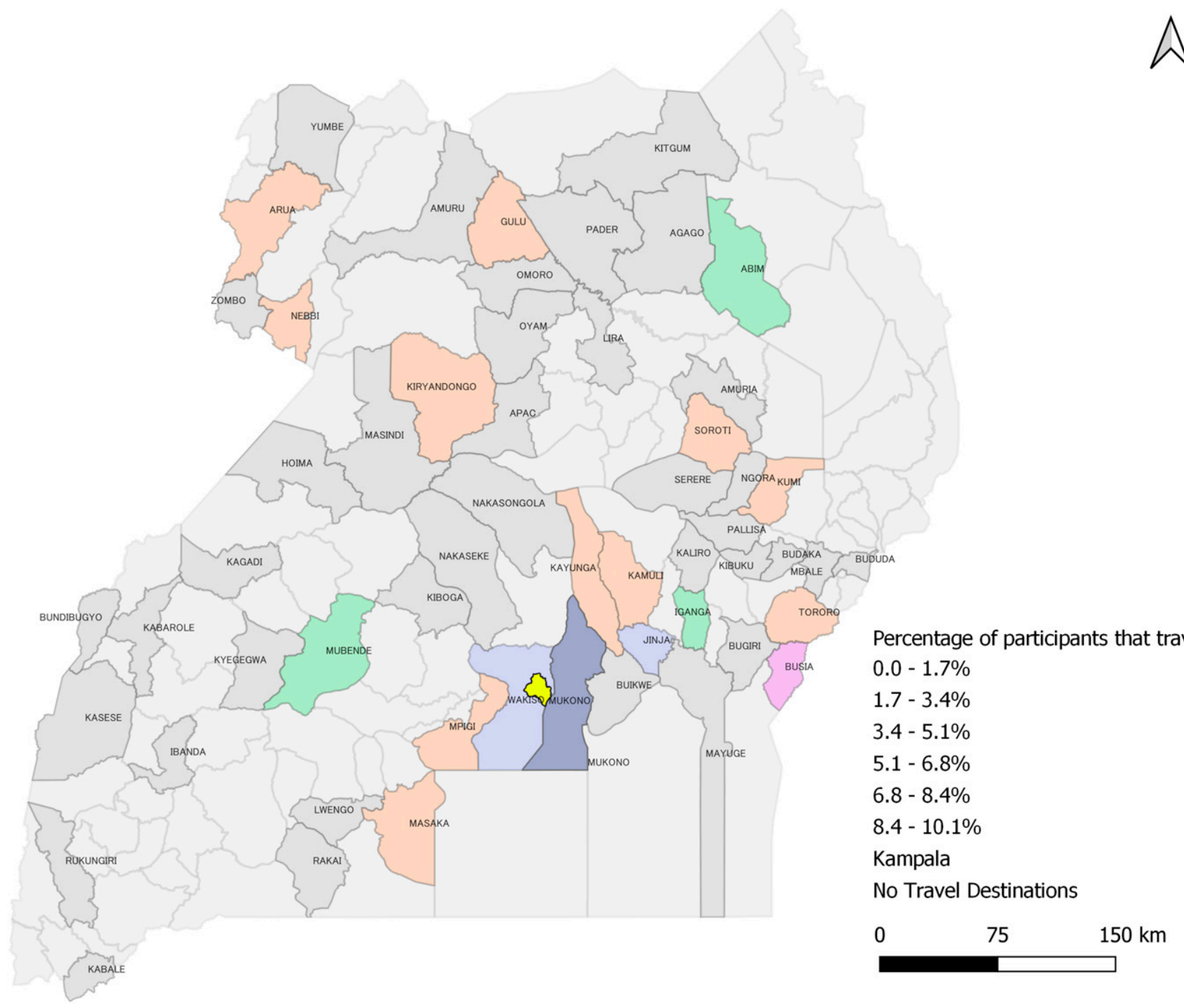

FIGURE 2. Map of Uganda showing destination of travel for study participants with a history of recent overnight travel out of Kampala. This figure appears in color at www.ajtmh.org.

We also found that, among travelers, engaging in outdoor activities was strongly associated with increased risk of malaria. Outdoor activities may increase exposure to mosquito bites and subsequent malaria, ${ }^{33-37}$ suggesting that use of personal protection measures to avoid outdoor exposure to malaria vectors during travel, including mosquito repellent creams and sprays, should be explored. Mosquito repellents have been used primarily for malaria prevention by travelers

TABLE 3

Recent overnight travel out of Kampala and the risk of malaria

\begin{tabular}{|c|c|c|c|c|c|}
\hline \multirow[b]{2}{*}{ Risk factor } & \multirow[b]{2}{*}{ Proportion testing positive for malaria, $n / N(\%)$} & \multicolumn{2}{|c|}{ Univariate analysis } & \multicolumn{2}{|c|}{ Multivariate analysis } \\
\hline & & $\mathrm{OR}(95 \% \mathrm{Cl})$ & $P$-value & OR $(95 \% \mathrm{Cl})$ & $P$-value \\
\hline \multicolumn{6}{|l|}{ Gender } \\
\hline Female & 75/329 (22.8) & Reference & & Reference & \\
\hline Male & $87 / 238(36.6)$ & $1.95(1.33-2.87)$ & 0.001 & $2.0(1.02-3.91)$ & 0.04 \\
\hline \multicolumn{6}{|l|}{ Place of residence within Kampala } \\
\hline Kampala central & $11 / 48(22.9)$ & Reference & & Reference & \\
\hline Kawempe division & $15 / 36(41.7)$ & $2.40(0.94-6.15)$ & 0.07 & $3.12(0.64-15.2)$ & 0.16 \\
\hline Makindye division & $20 / 61(32.8)$ & $1.43(0.61-3.38)$ & 0.41 & $2.14(0.47-9.81)$ & 0.33 \\
\hline Nakawa division & $114 / 409(27.9)$ & $1.23(0.61-2.47)$ & 0.57 & $1.59(0.51-4.93)$ & 0.42 \\
\hline Rubaga division & 2/13 (15.4) & $0.59(0.11-3.06)$ & 0.53 & $0.46(0.03-6.26)$ & 0.56 \\
\hline \multicolumn{6}{|l|}{ Recent overnight travel out of Kampala } \\
\hline No history of travel within 60 days & $35 / 409(8.6)$ & Reference & & Reference & \\
\hline History of travel within 60 days & $127 / 158(80.4)$ & $55.4(22.6-135.8)$ & $<0.001$ & $58.9(23.1-150.1)$ & $<0.001$ \\
\hline
\end{tabular}


TABLE 4

Risk factors for malaria in participants with a history of recent overnight travel out of Kampala

\begin{tabular}{|c|c|c|c|c|c|}
\hline \multirow[b]{2}{*}{ Risk factor } & \multirow[b]{2}{*}{ Proportion testing positive for malaria, $n / N(\%)$} & \multicolumn{2}{|c|}{ Univariate analysis } & \multicolumn{2}{|c|}{ Multivariate analysis } \\
\hline & & OR $(95 \% \mathrm{Cl})$ & $P$-value & OR $(95 \% \mathrm{Cl})$ & $P$-value \\
\hline \multicolumn{6}{|l|}{ Age category } \\
\hline 16 years or older & $67 / 95(70.5)$ & Reference & & Reference & \\
\hline Below 16 years & 60/63 (95.2) & $8.36(2.42-28.9)$ & 0.001 & $11.2(2.22-56.2)$ & 0.03 \\
\hline \multicolumn{6}{|l|}{ Gender } \\
\hline Female & $60 / 79(76.0)$ & Reference & & Reference & \\
\hline Male & $67 / 79(84.8)$ & $1.77(0.79-3.94)$ & 0.16 & $1.86(0.54-6.46)$ & 0.33 \\
\hline \multicolumn{6}{|l|}{ Destination of travel } \\
\hline IRS district & $3 / 13(23.1)$ & Reference & & Reference & \\
\hline Non-IRS district & $124 / 145(85.5)$ & $19.7(5.00-77.5)$ & $<0.001$ & $35.0(4.80-254.9)$ & $<0.001$ \\
\hline \multicolumn{6}{|c|}{ Duration of stay at destination of travel } \\
\hline Less than 10 days & $97 / 125(77.6)$ & Reference & & Reference & \\
\hline 10 days or more & $30 / 33(90.9)$ & $2.89(0.82-10.17)$ & 0.10 & $6.59(1.10-39.6)$ & 0.04 \\
\hline \multicolumn{6}{|c|}{ Reported ITN use during travel } \\
\hline Used an ITN & $20 / 40(50.0)$ & Reference & & Reference & \\
\hline Did not use an ITN & $107 / 118(90.7)$ & $9.73(4.05-23.4)$ & $<0.001$ & $30.1(6.37-142.7)$ & $<0.001$ \\
\hline \multicolumn{6}{|l|}{ Time of sleep during travel } \\
\hline After 9 PM & $84 / 109$ (77.1) & Reference & & Reference & \\
\hline 9 PM or earlier & 43/49 (87.8) & $2.13(0.81-5.59)$ & 0.12 & $0.91(0.18-4.68)$ & 0.91 \\
\hline \multicolumn{6}{|c|}{ Place for accommodation during travel } \\
\hline Other places & 16/21 (76.2) & Reference & & Reference & \\
\hline Relative's place & 111/137 (81.0) & $1.33(0.45-3.97)$ & 0.61 & $9.55(1.32-69.1)$ & 0.03 \\
\hline \multicolumn{6}{|c|}{ Activity carried out in the evening } \\
\hline Indoor activities & $91 / 120$ (75.8) & Reference & & Reference & \\
\hline Outdoor activities & $36 / 38(94.7)$ & $5.74(1.30-25.3)$ & 0.02 & $22.0(3.42-141.8)$ & 0.001 \\
\hline
\end{tabular}

from malaria non-endemic countries when traveling to malaria-endemic areas. ${ }^{38,39}$ Commonly used repellents, including N,N-diethyl-3-methylbenzamide, have good safety profiles. ${ }^{40}$ When used properly, repellents can provide protection for up to 6 hours. ${ }^{41}$ However, studies evaluating mass distribution of topical mosquito repellents have not shown an impact on malaria parasite prevalence because of poor compliance with daily use. ${ }^{42}$

Use of chemoprophylaxis, that is, regular administration of a drug to prevent malaria infection is a key component of malaria prevention in travelers. ${ }^{43,44}$ Chemoprophylaxis is recommended for travelers from malaria non-endemic countries to malaria-endemic regions, ${ }^{22}$ but the same practice might be beneficial for travelers to high transmission areas in Africa. Standard recommendations for malaria chemoprophylaxis are daily atovaquone-proguanil, daily doxycycline, or weekly mefloquine; primaquine and tafenoquine are also effective but require confirmation of normal glucose-6-phosphate dehydrogenase levels before use. ${ }^{43}$ Chemoprophylaxis should be initiated shortly before the trip begins and continued for 1-4 weeks after completion of travel, depending on the drug used. As with travelers from non-endemic countries, the use of chemoprophylaxis in addition to specific mosquito control measures is likely to provide substantial protection against malaria infection in travelers to high-risk areas of Africa.

Our study had some limitations. First, like all case-control studies, the study was prone to selection bias which can lead to mistaken inference. However, selection bias was minimized by selecting both cases and controls from the same outpatient department and using the same diagnostic algorithm for both groups. Second, we could not rule out recall bias concerning travel histories, especially because history of travel was defined over a 60-day period. It is possible that participants with malaria might have been more likely to recall or report travel as compared with those without malaria. Third, the case-control study design only allowed assessment of the association between overnight travel and malaria as odds ratios, and it was not possible to estimate the actual risk of malaria among travelers. Fourth, we did not capture data on behavioral or household factors that might affect malaria risk at participant's homes. It is possible that participants with higher riskassociated behaviors while traveling may practice those same patterns while at home, although risks would nonetheless be limited by the low malaria transmission intensity in Kampala. Last, the study was conducted over a period of 2 months and in only one hospital in Kampala, and results cannot be considered representative of risks at other times of the year, or for other locations. Nonetheless, the very high odds for malaria after travel strongly suggest that travel out of Kampala was responsible for the majority of malaria diagnosed in this urban setting.

In summary, malaria among Kampala residents was strongly associated with overnight travel, especially travel to high-risk districts of Uganda that were not receiving IRS. Among those who traveled, not using an ITN during travel, engaging in outdoor activities, staying with friends and relatives, and duration of travel for 10 days or more were associated with increased odds of malaria. Our findings suggest that personal malaria prevention measures, including use of ITNs, application of creams or sprays to prevent outdoor mosquito bites, and administration of chemoprophylaxis should be emphasized to protect individuals traveling overnight from low to high transmission regions of Africa.

Received March 13, 2020. Accepted for publication May 11, 2020.

Published online August 24, 2020.

Acknowledgments: We are grateful to the staff of Infectious Diseases Research Collaboration for administrative support and the study participants for their involvement in this study.

Financial support: This work was supported by the Fogarty International Centers of the National Institutes of Health under Award 
number D43TW010526 and D43TW7375. J. I. N. is supported by the Fogarty International Center (Emerging Global Leader Award grant number K43TW010365).

Disclaimer: The content of this report is solely the responsibility of the authors and does not necessarily represent the official views of the National Institutes of Health. The funders of this study did not participate in the study design, data collection, data analysis, and data interpretation or writing of findings.

Disclosures: All authors have not reported any conflict of interest and have submitted the ICMJE form for disclosure of potential conflict of interest.

Authors' addresses: Emmanuel Arinaitwe, Chris Drakeley, and Sarah Staedke, Department of Infectious and Tropical Diseases, London School of Hygiene and Tropical Medicine, London, United Kingdom, E-mails: earinaitwe@idrc-uganda.org, chris.drakeley@lshtm.ac.uk, and sarah.staedke@Ishtm.ac.uk. Arthur Mpimbaza, Joaniter I. Nankabirwa, and Moses R. Kamya, Makerere University and the Infectious Diseases Research Collaboration, Kampala, Uganda, E-mails: arthurwakg@yahoo.com, jnankabirwa@yahoo.co.uk, and mkamya@ infocom.co.ug. Victor Kamya and Alan Asiimwe, Infectious Diseases Research Collaboration, Kampala, Uganda, E-mail: vkamya@idrcuganda.org and aasiimwe@idrc-uganda.org. Julius K. Kuule, Uganda Malaria Research Centre, Ministry of Health, Kampala, Uganda, E-mail: jkkuule2000@yahoo.com.

\section{REFERENCES}

1. Bhatt $S$ et al., 2015. The effect of malaria control on Plasmodium falciparum in Africa between 2000 and 2015. Nature 526: 207-211.

2. Tatem AJ, Gething PW, Smith DL, Hay SI, 2013. Urbanization and the global malaria recession. Malar J 12: 133.

3. World Health Organization, 2019. World Malaria Report 2019. Geneva, Switzerland: World Health Organization.

4. Bukirwa H, Yau V, Kigozi R, Filler S, Quick L, Lugemwa M, Dissanayake G, Kamya M, Wabwire-Mangen F, Dorsey G, 2009. Assessing the impact of indoor residual spraying on malaria morbidity using a sentinel site surveillance system in western Uganda. Am J Trop Med Hyg 81: 611-614.

5. Kigozi $R$ et al., 2012. Indoor residual spraying of insecticide and malaria morbidity in a high transmission intensity area of Uganda. PLoS One 7: e42857.

6. Steinhardt LC et al., 2013. The effect of indoor residual spraying on malaria and anemia in a high-transmission area of northern Uganda. Am J Trop Med Hyg 88: 855-861.

7. Raouf $S$ et al., 2017. Resurgence of malaria following discontinuation of indoor residual spraying of insecticide in an area of Uganda with previously high-transmission intensity. Clin Infect Dis 65: 453-460.

8. Okullo AE, Matovu JKB, Ario AR, Opigo J, Wanzira H, Oguttu DW, Kalyango JN, 2017. Malaria incidence among children less than 5 years during and after cessation of indoor residual spraying in northern Uganda. Malar J 16: 319.

9. Uganda Bureau of Statistics (UBOS), 2015. Uganda Malaria Indicator Survey 2014-2015. Kampala, Uganda: Uganda Bureau of Statistics.

10. Cohen S, Mc Gl, Carrington S, 1961. Gamma-globulin and acquired immunity to human malaria. Nature 192: 733-737.

11. Nussenzweig RS, Vanderberg J, Most H, Orton C, 1967. Protective immunity produced by the injection of $\mathrm{x}$-irradiated sporozoites of Plasmodium berghei. Nature 216: 160-162.

12. Langhorne J, Ndungu FM, Sponaas AM, Marsh K, 2008. Immunity to malaria: more questions than answers. Nat Immunol 9: 725-732.

13. Ghani AC, Sutherland CJ, Riley EM, Drakeley CJ, Griffin JT, Gosling RD, Filipe JA, 2009. Loss of population levels of immunity to malaria as a result of exposure-reducing interventions: consequences for interpretation of disease trends. PLOS One 4: e4383.

14. Le Menach A, Tatem AJ, Cohen JM, Hay SI, Randell H, Patil AP, Smith DL, 2011. Travel risk, malaria importation and malaria transmission in Zanzibar. Sci Rep 1: 93.

15. Tatem AJ, Qiu Y, Smith DL, Sabot O, Ali AS, Moonen B, 2009. The use of mobile phone data for the estimation of the travel patterns and imported Plasmodium falciparum rates among Zanzibar residents. Malar J 8: 287.

16. Overgaard HJ, Reddy VP, Abaga S, Matias A, Reddy MR, Kulkarni V, Schwabe C, Segura L, Kleinschmidt I, Slotman MA, 2012. Malaria transmission after five years of vector control on Bioko Island, Equatorial Guinea. Parasit Vectors 5: 253.

17. Bradley J, Matias A, Schwabe C, Vargas D, Monti F, Nseng G, Kleinschmidt I, 2012. Increased risks of malaria due to limited residual life of insecticide and outdoor biting versus protection by combined use of nets and indoor residual spraying on Bioko Island, Equatorial Guinea. Malar J 11: 242.

18. Bradley J, Monti F, Rehman AM, Schwabe C, Vargas D, Garcia G, Hergott D, Riloha M, Kleinschmidt I, 2015. Infection importation: a key challenge to malaria elimination on Bioko Island, Equatorial Guinea. Malar J 14: 46.

19. Mathanga DP, Tembo AK, Mzilahowa T, Bauleni A, Mtimaukenena K, Taylor TE, Valim C, Walker ED, Wilson ML, 2016. Patterns and determinants of malaria risk in urban and peri-urban areas of Blantyre, Malawi. Malar J 15: 590.

20. Zinszer $\mathrm{K}$ et al., 2019. The impact of multiple rounds of indoor residual spraying on malaria incidence and haemoglobin levels in a high transmission setting. $J$ Infect Dis 221: 304-312.

21. Arinaitwe $E$ et al., 2019. Association between recent overnight travel and risk of malaria: a prospective cohort study at 3 sites in Uganda. Clin Infect Dis 68: 313-320.

22. World Health Organization, 2017. International Travel and Health. Available at: https://www.who.int/ith/2017-ith-chapter7.pdf? ua=1. Accessed February 5, 2020.

23. Ministry of Health Uganda, 2019. Uganda's Electronic Health Information System. Available at: https://hmis1.health.go.ug/ hmis2. Accessed February 5, 2020.

24. U.S. President's Malaria Initiative, 2020. The PMI VectorLink Project, Uganda. Available at: https://pmivectorlink.org/wherewe-work/uganda/. Accessed February 5, 2020.

25. Orish V, Afutu L, Ayodele O, Likaj L, Marinkovic A, Sanyaolu A, 2019. A 4-day incubation period of Plasmodium falciparum infection in a nonimmune patient in Ghana: a case report. Open Forum Infect Dis 6: ofy169.

26. Centers for Disease Control and Prevention, 2019. About Malaria. Available at: https://www.cdc.gov/malaria/about/disease.html. Accessed February 5, 2020

27. Warrell DA, Gilles HM, 2002. Essential Malariology. London, United Kingdom: Hodder Arnold.

28. Uganda Bureau of Statistics (UBOS), 2019. Uganda Malaria Indicator Survey 2018-2019. Kampala, Uganda: Uganda Bureau of Statistics.

29. Baragatti M, Fournet F, Henry MC, Assi S, Ouedraogo H, Rogier C, Salem G, 2009. Social and environmental malaria risk factors in urban areas of Ouagadougou, Burkina Faso. Malar J 8: 13.

30. Maharaj R, Moonasar D, Baltazar C, Kunene S, Morris N, 2016. Sustaining control: lessons from the Lubombo spatial development initiative in southern Africa. Malar J 15: 409.

31. Moonasar D, Nuthulaganti T, Kruger PS, Mabuza A, Rasiswi ES, Benson FG, Maharaj R, 2012. Malaria control in South Africa 2000-2010: beyond MDG6. Malar J 11: 294.

32. Monroe A, Harvey SA, Lam Y, Muhangi D, Loll D, Kabali AT, Weber R, 2014. "People will say that I am proud": a qualitative study of barriers to bed net use away from home in four Ugandan districts. Malar J 13: 82.

33. Chirebvu E, Chimbari MJ, Ngwenya BN, 2014. Assessment of risk factors associated with malaria transmission in tubu village, northern Botswana. Malar Res Treat 2014: 1-10.

34. Thomsen EK, Koimbu G, Pulford J, Jamea-Maiasa S, Ura Y, Keven JB, Siba PM, Mueller I, Hetzel MW, Reimer LJ, 2017. Mosquito behavior change after distribution of bednets results in decreased protection against malaria exposure. $J$ Infect Dis 215: 790-797.

35. Moshi IR, Manderson L, Ngowo HS, Mlacha YP, Okumu FO, Mnyone LL, 2018. Outdoor malaria transmission risks and social life: a qualitative study in south-eastern Tanzania. Malar $J$ 17: 397

36. Finda MF et al., 2019. Linking human behaviours and malaria vector biting risk in south-eastern Tanzania. PLOS One 14: e0217414. 
37. Sherrard-Smith E et al., 2019. Mosquito feeding behavior and how it influences residual malaria transmission across Africa. Proc Natl Acad Sci U S A 116: 15086-15095.

38. Yap HH, Jahangir K, Zairi J, 2000. Field efficacy of four insect repellent products against vector mosquitoes in a tropical environment. J Am Mosq Control Assoc 16: 241-244.

39. Islam J, Zaman K, Duarah S, Raju PS, Chattopadhyay P, 2017. Mosquito repellents: an insight into the chronological perspectives and novel discoveries. Acta Trop 167: 216-230.

40. McGready R, Hamilton KA, Simpson JA, Cho T, Luxemburger C, Edwards R, Looareesuwan S, White NJ, Nosten F, Lindsay SW, 2001. Safety of the insect repellent N,N-diethyl-M-toluamide (DEET) in pregnancy. Am J Trop Med Hyg 65: 285-289.
41. Goodyer LI, Croft AM, Frances SP, Hill N, Moore SJ, Onyango SP, Debboun M, 2010. Expert review of the evidence base for arthropod bite avoidance. J Travel Med 17: 182-192.

42. Sluydts V et al., 2016. Efficacy of topical mosquito repellent (picaridin) plus long-lasting insecticidal nets versus long-lasting insecticidal nets alone for control of malaria: a cluster randomised controlled trial. Lancet Infect Dis 16: 1169-1177.

43. Centers for Disease Control and Prevention, 2019. Malaria and Travelers for U.S. Residents. Available at: https://www.cdc.gov/ malaria/travelers/index.html. Accessed February 5, 2020.

44. Public Health England, 2019. Malaria Prevention Guidelines for Travellers from the UK. Available at: https://www.gov.uk/ government/publications/malaria-prevention-guidelines-fortravellers-from-the-uk. Accessed February 5, 2020. 